\title{
MINIMAXNESS AND FINITENESS PROPERTIES OF FORMAL LOCAL COHOMOLOGY MODULES
}

\author{
SHAHRAM REZAEI
}

\begin{abstract}
Let $\mathfrak{a}$ be an ideal of local ring $(R, \mathrm{~m})$ and $M$ a finitely generated $R$-module and $n$ an integer. We prove some results concerning minimaxness and finiteness of formal local cohomology modules. We discuss the maximum and minimum integers such that $\mathfrak{F}_{a}^{i}(M)$ is minimax and also we obtain the maximum and minimum integers such that $\mathfrak{F}_{\mathfrak{a}}^{i}(M)$ is finitely gnerated.
\end{abstract}

\section{Introduction}

Throughout this paper, $R$ is a commutative Noetherian ring with identity, a is an ideal of $R$ and $M$ is an $R$-module. Recall that the $i$-th local cohomology module of $M$ with respect to $\mathfrak{a}$ is denoted by $\mathrm{H}_{\mathfrak{a}}^{i}(M)$. For basic facts about commutative algebra see [4], [6]; for local cohomology refer to [3]. Let a be an ideal of a local ring $(R, \mathrm{~m})$ and $M$ a finitely generated $R$-module. For each $i \geq 0 ; \mathfrak{F}_{\mathfrak{a}}^{i}(M):={\underset{\lim }{r}}_{\mathrm{H}_{m}}^{i}\left(M / \mathfrak{a}^{n} M\right)$ is called the $i$-th formal local cohomology of $M$ with respect to $\mathfrak{a}$.

The basic properties of formal local cohomology modules are found in [7], [1], [5] and [2].

Recall that an $R$-module $M$ is called minimax, if there is a finite submodule $N$ of $M$, such that $M / N$ is Artinian. The class of minimax modules was introduced by Zöschinger [10], and he has given in [10, 11] many equivalent conditions for a module to be minimax. The class of minimax modules includes all finite and all Artinian modules. Moreover it is closed under taking submodules, quotients and extensions, i.e., it is a Serre subcategory of the category of $R$-modules.

In this paper we investigate some Minimaxness and Finiteness properties of formal local cohomology modules. At first we obtain a result about cosupport

2000 Mathematics Subject Classification. 13D45, 13 E99.

Key words and phrases. formal local cohomology, local cohomology.

Received June 17, 2014; revised November 19, 2014. 
of minimax formal local cohomology modules and then we determine the least and the largest integers $i$ such that $\widetilde{F}_{\mathfrak{a}}^{i}(M)$ is minimax. Also we investigate the relation between Finiteness and cosupport of formal local cohomology modules. We will get that (see Theorem 2.8):

$$
\begin{aligned}
\inf \{i & \left.\in \mathbf{N}_{0}: \mathfrak{F}_{\mathfrak{a}}^{i}(M) \text { is not finitely generated }\right\} \\
& =\inf \left\{i \in \mathbf{N}_{0}: \operatorname{Cosupp}_{R}\left(\mathfrak{F}_{\mathfrak{a}}^{i}(M)\right) \nsubseteq\{\mathfrak{m}\}\right\}
\end{aligned}
$$

and by Theorem 2.10:

$$
\begin{aligned}
\sup \left\{i \in \mathbf{N}_{0}: \mathfrak{F}_{\mathfrak{a}}^{i}(M) \neq 0\right\}=\sup \left\{i \in \mathbf{N}_{0}: \mathfrak{F}_{\mathfrak{a}}^{i}(M) \text { is not finitely generated }\right\} \\
\quad=\sup \left\{i \in \mathbf{N}_{0}: \operatorname{Coass}_{R}\left(\mathfrak{F}_{\mathfrak{a}}^{i}(M)\right) \nsubseteq\{\mathfrak{m}\}\right\}
\end{aligned}
$$

\section{Finiteness of formal local cohomology modules}

We first recall the concept of coassociated primes and cosupport of an $R$-module $M$. A module is called cocyclic if it is a submodule of $E(R / \mathrm{m})$ for some maximal ideal $\mathrm{m}$ of $R$. A prime ideal $\mathfrak{p}$ is called coassociated to a non-zero $R$-module $M$ if there is a cocyclic homomorphic image $T$ of $M$ with $\mathfrak{p}=\operatorname{Ann}_{R} T$ [8]. The set of coassociated primes of $M$ is denoted by $\operatorname{Coass}_{R}(M)$. Also, Yassemi [8] defined the cosupport of an $R$-module $M$, denoted by $\operatorname{Cosupp}_{R}(M)$, to be the set of primes $\mathfrak{p}$ such that there exists a cocyclic homomorphic image $L$ of $M$ with $\operatorname{Ann}_{R}(L) \subseteq \mathfrak{p}$. In [8] we can see that $\operatorname{Coass}_{R}(M) \subseteq \operatorname{Cosupp}_{R}(M)$ and every minimal element of the set $\operatorname{Cosupp}_{R}(M)$ belongs to $\operatorname{Coass}_{R}(M)$.

The following lemma is used in the sequel.

LEMMA 2.1. Let $\mathfrak{a}$ be an ideal of local ring $(R, \mathfrak{m})$ and $M$ an $R$-module. If $\mathfrak{a}^{k} M=0$ for some $k \in \mathbf{N}$, then $\mathfrak{F}_{\mathfrak{a}}^{i}(M) \cong \mathrm{H}_{\mathfrak{m}}^{i}(M)$. Therefore $\mathfrak{F}_{\mathfrak{a}}^{i}(M)$ is Artinian for all $i \geq 0$ and so it is also minimax.

Proof. It is clear that $\mathfrak{F}_{\mathfrak{a}}^{i}(M) \cong \lim _{\longleftarrow} \mathrm{H}_{m}^{i}\left(M / \mathfrak{a}^{n} M\right) \cong \mathrm{H}_{m}^{i}(M)$. But by [3, Theorem 7.1.3] $\mathrm{H}_{\mathfrak{m}}^{i}(M)$ is Artinian for all $i \geq 0$ and so the proof is complete.

THEOREM 2.2. Let $\mathfrak{a}$ be an ideal of a local ring $(R, \mathrm{~m})$ and $M$ a finitely generated $R$-module. Let $i$ be a natural number. If $\mathfrak{F}_{\mathfrak{a}}^{i}(M)$ is minimax, then $\operatorname{Cosupp}_{R} \mathfrak{F}_{\mathfrak{a}}^{i}(M) \subseteq V(\mathfrak{a})$.

Proof. Since $\mathfrak{F}_{\mathfrak{a}}^{i}(M)$ is minimax, there exists a finitely generated submodule $N$ of $\mathfrak{F}_{\mathfrak{a}}^{i}(M)$ such that $\mathfrak{F}_{\mathfrak{a}}^{i}(M) / N$ is Artinian. If in [2, Theorem 2.3], we replace $\mathfrak{F}_{\mathfrak{a}}^{i}(M)$ with $\mathfrak{F}_{\mathfrak{a}}^{i}(M) / N$ then with small changes in its proof we can 
deduce that $\operatorname{Att}_{R} \mathfrak{F}_{\mathfrak{a}}^{i}(M) / N \subseteq V(\mathfrak{a})$. On the other hand by [8, Theorem 1.14] $\operatorname{Coass}_{R} \mathfrak{F}_{\mathfrak{a}}^{i}(M) / N=\operatorname{Att}_{R} \mathfrak{F}_{\mathfrak{a}}^{i}(M) / N$. Hence $\operatorname{Coass}_{R} \mathfrak{F}_{\mathfrak{a}}^{i}(M) / N \subseteq V(\mathfrak{a})$ and so $\operatorname{Cosupp}_{R} \mathfrak{F}_{\mathfrak{a}}^{i}(M) / N \subseteq V(\mathfrak{a})$. Since $N$ is finitely generated $\operatorname{Cosupp}_{R} N \subseteq V(\mathfrak{m})$ by [8, Theorem 2.10]. Thus $\operatorname{Cosupp}_{R} \mathfrak{F}_{\mathfrak{a}}^{i}(M) \subseteq V(\mathfrak{a})$ by [8, Theorem 2.7], as required.

We need the following Lemma in the proof of the Next Theorem.

Lemma 2.3. Let $\mathfrak{a}$ be an ideal of a local ring $(R, \mathfrak{m})$ and $M$ a finitely generated R-module. Then $\mathfrak{F}_{\mathfrak{a}}^{0}(M) / \mathfrak{a}^{k} \cdot \mathfrak{F}_{\mathfrak{a}}^{0}(M)$ is Artinian for all $k \in \mathbf{N}$.

Proof. By [1, Theorem 3.8] $\mathfrak{F}_{\mathfrak{a}^{k}}^{0}(M) / \mathfrak{a}^{k} \cdot \mathfrak{F}_{\mathfrak{a}^{k}}^{0}(M)$ is Artinian for all $k \in \mathbf{N}$. But $\mathfrak{F}_{\mathfrak{a}}^{0}(M) \simeq \mathfrak{F}_{\mathfrak{a}^{k}}^{0}(M)$ by [7, Lemma 3.8] and so we get the result.

THEOREM 2.4. Let $\mathfrak{a}$ be an ideal of a local ring $(R, \mathrm{~m})$ and $M$ a finitely generated $R$-module, and let $n \in \mathbf{N}$. Then the following statements are equivalent:

i) $\mathfrak{F}_{\mathfrak{a}}^{i}(M)$ is minimax for all $i<n$.

ii) $\operatorname{Cosupp}_{R}\left(\mathfrak{F}_{\mathfrak{a}}^{i}(M)\right) \subseteq V(\mathfrak{a})$ for all $i<n$.

Proof. i) $\Rightarrow$ ii): By Theorem 2.2.

ii) $\Rightarrow$ i): We use induction on $n$. Since $\operatorname{Coass}_{R}\left(\mathfrak{F}_{\mathfrak{a}}^{i}(M)\right) \subseteq \operatorname{Cosupp}_{R}\left(\mathfrak{F}_{\mathfrak{a}}^{i}(M)\right)$ $\subseteq V(\mathfrak{a})$, by $\left[9\right.$, Satz 2.4] there exists an integer $k \geq 0$ such that $\mathfrak{a}^{k} \mathfrak{F}_{\mathfrak{a}}^{i}(M)$ is finitely generated. By Lemma 2.3, $\mathfrak{F}_{\mathfrak{a}}^{0}(M) / \mathfrak{a}^{k} \mathfrak{F}_{\mathfrak{a}}^{0}(M)$ is Artinian and so $\mathfrak{F}_{\mathfrak{a}}^{0}(M)$ is minimax.

Now suppose, inductively, that $n>0$ and we have established the result for smaller values of $n$. Thus $\mathfrak{F}_{\mathfrak{a}}^{i}(M)$ is minimax for all $i \leq n-2$. It is enough to show that $\mathfrak{F}_{\mathfrak{a}}^{n-1}(M)$ is minimax. By [7, Theorem 3.11], the short exact sequence

$$
0 \rightarrow \Gamma_{\mathfrak{a}}(M) \rightarrow M \rightarrow M / \Gamma_{\mathfrak{a}}(M) \rightarrow 0
$$

implies the long exact sequence

$$
\cdots \rightarrow \mathfrak{F}_{\mathfrak{a}}^{i-1}\left(\Gamma_{\mathfrak{a}}(M)\right) \rightarrow \mathfrak{F}_{\mathfrak{a}}^{i-1}(M) \rightarrow \mathfrak{F}_{\mathfrak{a}}^{i-1}\left(M / \Gamma_{\mathfrak{a}}(M)\right) \rightarrow \mathfrak{F}_{\mathfrak{a}}^{i}\left(\Gamma_{\mathfrak{a}}(M)\right) \rightarrow \cdots
$$

Since by Lemma $2.1, \mathfrak{F}_{\mathfrak{a}}^{i}\left(\Gamma_{\mathfrak{a}}(M)\right)$ is minimax for all $i \geq 0$, using the above long exact sequence, we can see that $\mathfrak{F}_{\mathfrak{a}}^{i}(M)$ is minimax if and only if $\mathfrak{F}_{\mathfrak{a}}^{i}\left(M / \Gamma_{\mathfrak{a}}(M)\right)$ is minimax for all $i \geq 0$. On the other hand, since $\mathfrak{F}_{\mathfrak{a}}^{i}\left(\Gamma_{\mathfrak{a}}(M)\right)$ is minimax for all $i \geq 0, \operatorname{Cosupp}_{R} \mathfrak{F}_{\mathfrak{a}}^{i}\left(\Gamma_{\mathfrak{a}}(M)\right) \subseteq V(\mathfrak{a})$ for all $i \geq 0$ by Theorem 2.2. Now the above long exact sequance and our assumption that $\operatorname{Cosupp}_{R} \mathfrak{F}_{\mathfrak{a}}^{i}(M) \subseteq V(\mathfrak{a})$ for all $i<n$ imply that Cosupp $\mathfrak{F}_{\mathfrak{a}}^{i}\left(M / \Gamma_{\mathfrak{a}}(M)\right) \subseteq V(\mathfrak{a})$ for all $i<n$. Therefore we can and do assume that $M$ is an a-torsion-free $R$-module.

By $[3,2.1 .1$ (ii)], a contains an element $r$ which is a non-zerodivisor on $M$. Since $\operatorname{Cosupp}_{R} \mathfrak{F}_{\mathfrak{a}}^{n-1}(M) \subseteq V(\mathfrak{a})$, by [9, Satz 2.4] there is an integer $k \geq 1$ such that $r^{k} \mathfrak{F}_{\mathfrak{a}}^{n-1}(M)$ is finitely generated. The short exact sequence

$$
0 \rightarrow M \stackrel{r^{k}}{\rightarrow} M \rightarrow M / r^{k} M \rightarrow 0
$$


induces a long exact sequence

$$
\begin{aligned}
0 & \rightarrow \mathfrak{F}_{\mathfrak{a}}^{0}(M) \stackrel{r^{k}}{\rightarrow} \mathfrak{F}_{\mathfrak{a}}^{0}(M) \rightarrow \mathfrak{F}_{\mathfrak{a}}^{0}\left(M / r^{k} M\right) \rightarrow \cdots \rightarrow \mathfrak{F}_{\mathfrak{a}}^{i}(M) \\
& \stackrel{r^{k}}{\rightarrow} \mathfrak{F}_{\mathfrak{a}}^{i}(M) \rightarrow \mathfrak{F}_{\mathfrak{a}}^{i}\left(M / r^{k} M\right) \rightarrow \cdots
\end{aligned}
$$

From this long exact sequence, it turns out that $\operatorname{Cosupp}_{R} \mathfrak{F}_{\mathfrak{a}}^{i}\left(M / r^{k} M\right) \subseteq V(\mathfrak{a})$ for all $i<n-1$. Hence, by the inductive hypothesis, $\mathfrak{F}_{\mathfrak{a}}^{i}\left(M / r^{k} M\right)$ is minimax for all $i<n-1$, and so $\mathfrak{F}_{\mathfrak{a}}^{n-2}\left(M / r^{k} M\right)$ is minimax. Since $r^{k} \mathfrak{F}_{\mathfrak{a}}^{n-1}(M)$ is finitely generated and so is minimax, the above long exact sequence implies that $\mathfrak{F}_{\mathfrak{a}}^{n-2}\left(M / r^{k} M\right) \rightarrow \mathfrak{F}_{\mathfrak{a}}^{n-1}(M) \rightarrow r^{k} \mathfrak{F}_{\mathfrak{a}}^{n-1}(M)$ is exact. Thus $\mathfrak{F}_{\mathfrak{a}}^{n-1}(M)$ is minimax, as required.

THEOREM 2.5. Let $\mathfrak{a}$ be an ideal of a local ring $(R, \mathrm{~m})$ and $M$ a finitely generated $R$-module, and let $t \in \mathbf{N}$. Then the following statements are equivalent:

(i) $\mathfrak{F}_{\mathfrak{a}}^{j}(M)$ is minimax for all $j>t$.

(ii) $\operatorname{Cosupp}_{R} \mathfrak{F}_{\mathfrak{a}}^{j}(M) \subseteq V(\mathfrak{a})$ for all $j>t$.

Proof. (i) $\Rightarrow$ (ii): By Theorem 2.2.

(ii) $\Rightarrow$ (i): We argue by induction on $n:=\operatorname{dim} M$. If $n=0$, then $\mathfrak{F}_{\mathfrak{a}}^{i}(M)=0$ for all $i>0$, so that the result has been proved in this case. Now assume, inductively, that $n>0$ and that the result has been proved for all $R$-modules of dimensions smaller than $n$. By an argument analogue to that used in the proof of Theorem 2.4, we can and do assume that $M$ is an a-torsion-free $R$-module. By [3, 2.1.1 (ii)], a contains an element $r$ which is a non-zerodivisor on $M$. Let $j>t$ be an integer. By assumption and [9, Satz 2.4] there exists an integer $u_{j}$ such that $\mathfrak{a}^{u_{j}} \mathfrak{F}_{\mathfrak{a}}^{j}(M)$ is finitely generated. But $\mathfrak{F}_{\mathfrak{a}}^{i}(M)=0$ for all $i>\operatorname{dim} M$. Thus we can find an integer $u$ such that $\mathfrak{a}^{u} \mathfrak{F}_{\mathfrak{a}}^{j}(M)$ is finitely generated for all $j>t$. The exact sequence

$$
0 \rightarrow M \stackrel{r^{u}}{\rightarrow} M \rightarrow M / r^{u} M \rightarrow 0
$$

induces a long exact sequence of formal local cohomology modules

$$
\begin{aligned}
0 & \rightarrow \mathfrak{F}_{\mathfrak{a}}^{0}(M) \stackrel{r^{u}}{\rightarrow} \mathfrak{F}_{\mathfrak{a}}^{0}(M) \rightarrow \mathfrak{F}_{\mathfrak{a}}^{0}\left(M / r^{u} M\right) \rightarrow \cdots \rightarrow \mathfrak{F}_{\mathfrak{a}}^{i}(M) \\
& \stackrel{r^{u}}{\rightarrow} \mathfrak{F}_{\mathfrak{a}}^{i}(M) \rightarrow \mathfrak{F}_{\mathfrak{a}}^{i}\left(M / r^{u} M\right) \rightarrow \cdots
\end{aligned}
$$

From this long exact sequence, we get $\operatorname{Cosupp}_{R} \mathfrak{F}_{\mathfrak{a}}^{j}\left(M / r^{u} M\right) \subseteq V(\mathfrak{a})$ for all $j>t$. Since $\operatorname{dim}\left(M / r^{u} M\right)=n-1$, it follows from the inductive hypothesis that $\mathfrak{F}_{\mathfrak{a}}^{j}\left(M / r^{u} M\right)$ is minimax for all $j>t$. The exact sequence

$$
0 \rightarrow M \stackrel{r^{u}}{\rightarrow} M \rightarrow M / r^{u} M \rightarrow 0
$$

provides the following exact sequence

$$
\rightarrow r^{u} \mathfrak{F}_{\mathfrak{a}}^{j}(M) \rightarrow \mathfrak{F}_{\mathfrak{a}}^{j}(M) \rightarrow \mathfrak{F}_{\mathfrak{a}}^{j}\left(M / r^{u} M\right) \rightarrow \cdots,
$$


for all $j>t$. Since $\mathfrak{a}^{u} \mathfrak{F}_{\mathfrak{a}}^{j}(M)$ is finitely generated for all $j>t, r^{u} \mathfrak{F}_{\mathfrak{a}}^{j}(M)$ is minimax for all $j>t$. Thus $\mathfrak{F}_{\mathfrak{a}}^{j}(M)$ is minimax for all $j>t$. This completes the proof.

THEOREM 2.6. Let $\mathfrak{a}$ be an ideal of a local ring $(R, \mathfrak{m})$ and $M$ a finitely generated $R$-module, and let $t \in \mathbf{N}$. If $\mathfrak{F}_{\mathfrak{a}}^{i}(M)$ is minimax for all $i>t$ then $\operatorname{Supp}_{R} \mathfrak{F}_{\mathfrak{a}}^{i}(M) \cap V(\mathfrak{a}) \subseteq\{\mathfrak{m}\}$ for all $i>t$.

Proof. We use induction on $n:=\operatorname{dim} M$. If $n=0$, then $\mathfrak{F}_{\mathfrak{a}}^{i}(M)=0$ for all $i>0$, so that the result has been proved in this case. Now assume, inductively, that $n>0$ and that the result has been proved for all $R$-modules of dimensions smaller than $n$.

The short exact sequence

$$
0 \rightarrow \Gamma_{\mathfrak{a}}(M) \rightarrow M \rightarrow M / \Gamma_{\mathfrak{a}}(M) \rightarrow 0
$$

induces a long exact sequence

$$
\cdots \rightarrow \mathfrak{F}_{\mathfrak{a}}^{i}\left(\Gamma_{\mathfrak{a}}(M)\right) \rightarrow \mathfrak{F}_{\mathfrak{a}}^{i}(M) \rightarrow \mathfrak{F}_{\mathfrak{a}}^{i}\left(M / \Gamma_{\mathfrak{a}}(M)\right) \rightarrow \mathfrak{F}_{\mathfrak{a}}^{i+1}\left(\Gamma_{\mathfrak{a}}(M)\right) \rightarrow \cdots
$$

Since $\mathfrak{F}_{\mathfrak{a}}^{i}\left(\Gamma_{\mathfrak{a}}(M)\right)$ is Artinian for all $i \geq 0$ we obtain the following exact sequance for all prime ideal $\mathfrak{p} \varsubsetneqq \mathrm{m}$ :

$$
0 \rightarrow\left(\mathfrak{F}_{\mathfrak{a}}^{i}(M)\right)_{\mathfrak{p}} \rightarrow\left(\mathfrak{F}_{\mathfrak{a}}^{i}\left(M / \Gamma_{\mathfrak{a}}(M)\right)\right)_{\mathfrak{p}} \rightarrow 0
$$

From the above exact sequence we conclude that $\operatorname{Supp}_{R} \mathfrak{F}_{\mathfrak{a}}^{i}(M) \cap V(\mathfrak{a}) \subseteq$ $\{\mathfrak{m}\}$ if and only if $\operatorname{Supp}_{R} \mathfrak{F}_{\mathfrak{a}}^{i}\left(M / \Gamma_{\mathfrak{a}}(M)\right) \cap V(\mathfrak{a}) \subseteq\{\mathfrak{m}\}$. Hence we can and do assume that $M$ is an a-torsion-free $R$-module. Thus, there exists an element $x \in \mathfrak{a}$ which is a non-zerodivisor on $M$. Now the exact sequence

$$
0 \rightarrow M \stackrel{x}{\rightarrow} M \rightarrow M / x M \rightarrow 0
$$

induces a long exact sequence

$$
\cdots \rightarrow \mathfrak{F}_{\mathfrak{a}}^{i-1}(M / x M) \rightarrow \mathfrak{F}_{\mathfrak{a}}^{i}(M) \rightarrow \mathfrak{F}_{\mathfrak{a}}^{i}(M) \rightarrow \mathfrak{F}_{\mathfrak{a}}^{i}(M / x M) \rightarrow \cdots
$$

From the above exact sequance we see that $\mathfrak{F}_{\mathfrak{a}}^{i}(M / x M)$ is minimax for all $i>t$. But $\operatorname{dim}(M / x M)=n-1$ and so from the inductive hypothesis $\operatorname{Supp}_{R}\left(\mathfrak{F}_{\mathfrak{a}}^{i}(M / x M)\right) \cap V(\mathfrak{a}) \subseteq\{\mathfrak{m}\}$ for all $i>t$. Thus if $\mathfrak{p}$ is a prime ideal such that $\mathfrak{a} \subseteq \mathfrak{p} \subsetneq \mathfrak{m}$ then $\left(\mathfrak{F}_{\mathfrak{a}}^{i}(M / x M)\right)_{\mathfrak{p}}=0$ for all $i>t$. Hence we get the following exact sequance:

$$
\rightarrow\left(\mathfrak{F}_{\mathfrak{a}}^{i}(M)\right)_{\mathfrak{p}} \stackrel{x / 1}{\rightarrow}\left(\mathfrak{F}_{\mathfrak{a}}^{i}(M)\right)_{\mathfrak{p}} \rightarrow 0 .
$$

Let $i>t$ be an integer. Since $\mathfrak{F}_{\mathfrak{a}}^{i}(M)$ is minimax, there exists a finitely generated submodule $N$ of $\mathfrak{F}_{\mathfrak{a}}^{i}(M)$ such that $\mathfrak{F}_{\mathfrak{a}}^{i}(M) / N$ is Artinian. Thus $\left(\mathfrak{F}_{\mathfrak{a}}^{i}(M) / N\right)_{\mathfrak{p}}=0$ and so $\left(\mathfrak{F}_{\mathfrak{a}}^{i}(M)\right)_{\mathfrak{p}}$ is a finitely generated $R$-module. Hence $\left(\mathfrak{F}_{\mathfrak{a}}^{i}(M)\right)_{\mathfrak{p}}$ is finitely generated for all $i>t$. Now the above exact sequance and Nakayama Lemma imply that $\left(\mathfrak{F}_{\mathfrak{a}}^{i}(M)\right)_{\mathfrak{p}}=0$. Therefore $\mathfrak{p} \notin \operatorname{Supp}_{R}\left(\mathfrak{F}_{\mathfrak{a}}^{i}(M)\right)$ for all $\mathfrak{a} \subseteq \mathfrak{p} \subsetneq \mathrm{m}$. This completes the proof. 
COROllary 2.7. Let $\mathfrak{a}$ be an ideal of a local ring $(R, \mathrm{~m})$ and $M$ a finitely generated $R$-module, and let $t \in \mathbf{N}$. If $\mathfrak{F}_{\mathfrak{a}}^{i}(M)$ is minimax for all $i>t$ then $\operatorname{Hom}_{R}\left(R / \mathfrak{a}, \mathfrak{F}_{\mathfrak{a}}^{i}(M)\right)$ is Artinian for all $i>t$.

Proof. By Theorem 2.6, $\operatorname{Ass}_{R}\left(\operatorname{Hom}_{R}\left(R / \mathfrak{a}, \mathfrak{F}_{\mathfrak{a}}^{i}(M)\right)\right)=\operatorname{Ass}_{R} \mathfrak{F}_{\mathfrak{a}}^{i}(M) \cap V(\mathfrak{a}) \subseteq$ $\{\mathfrak{m}\}$ for all $i>t$. But $\operatorname{Hom}_{R}\left(R / \mathfrak{a}, \mathfrak{F}_{\mathfrak{a}}^{i}(M)\right) \simeq\left(0::_{\widetilde{x}_{a}^{i}(M)} \mathfrak{a}\right)$ is isomorphic to a submodule of $\mathfrak{F}_{\mathfrak{a}}^{i}(M)$ and so is minimax for all $i>t$. Now it is easy to see that, by the definition of minimax modules, $\operatorname{Hom}_{R}\left(R / \mathfrak{a}, \mathfrak{F}_{\mathfrak{a}}^{i}(M)\right)$ is Artinian for all $i>t$, as required.

THEOREM 2.8. Let $\mathfrak{a}$ be an ideal of a local ring $(R, \mathrm{~m})$ and $M$ a finitely generated $R$-module, and let $n \in \mathbf{N}$. Then the following statements are equivalent:

i) $\mathfrak{F}_{\mathfrak{a}}^{i}(M)$ is finitely generated for all $i<n$.

ii) $\operatorname{Cosupp}_{R}\left(\mathfrak{F}_{\mathfrak{a}}^{i}(M)\right) \subseteq\{\mathfrak{m}\}$ for all $i<n$.

Proof. i) $\Rightarrow$ ii): By [8, Theorem 2.10].

ii) $\Rightarrow$ i): Since $\operatorname{Cosupp}_{R} \mathfrak{F}_{\mathfrak{a}}^{i}(M) \subseteq\{\mathfrak{m}\}$ for all $i<n, \operatorname{Cosupp}_{R} \mathfrak{F}_{\mathfrak{a}}^{i}(M) \subseteq V(\mathfrak{a})$ for all $i<n$ and so $\mathfrak{F}_{\mathfrak{a}}^{i}(M)$ is minimax for all $i<n$ by Theorem 2.4. Let $i<n$ be an integer. Then there exists a finitely generated submodule $N$ such that $\mathfrak{F}_{\mathfrak{a}}^{i}(M) / N$ is Artinian. Hence $\operatorname{Att}_{R} \mathfrak{F}_{\mathfrak{a}}^{i}(M) / N=\operatorname{Coass}_{R} \mathfrak{F}_{\mathfrak{a}}^{i}(M) / N$. But $\operatorname{Coass}_{R} \mathfrak{F}_{\mathfrak{a}}^{i}(M) / N \subseteq \operatorname{Cosupp}_{R} \mathfrak{F}_{\mathfrak{a}}^{i}(M) / N \subseteq \operatorname{Cosupp}_{R} \mathfrak{F}_{\mathfrak{a}}^{i}(M) \subseteq\{\mathfrak{m}\}$ and so $\operatorname{Att}_{R} \mathfrak{F}_{\mathfrak{a}}^{i}(M) / N \subseteq\{\mathfrak{m}\}$. Now [3, 7.2.12] implies that $\mathfrak{F}_{\mathfrak{a}}^{i}(M) / N$ is finitely generated. Since $N$ is finitely generated we conclude that $\mathfrak{F}_{\mathfrak{a}}^{i}(M)$ is finitely generated for all $i<n$, as required.

Lemma 2.9. Let $(R, \mathrm{~m})$ be a local ring and $M$ be an $R$-module such that $\operatorname{Coass}_{R}(M) \subseteq\{\mathrm{m}\}$. Then $\operatorname{Hom}_{R}\left(R_{x}, M\right)=0$ for all $x \in \mathrm{m}$.

Proof. Since $\operatorname{Coass}_{R}(M) \subseteq\{\mathrm{m}\}$, there is an integer $t \geq 1$ such that $\mathrm{m}^{t} M$ is finitely generated. Now if $f \in \operatorname{Hom}_{R}\left(R_{x}, M\right)$ then $f\left(1 / x^{n}\right)=x^{k} x^{t} f\left(1 / x^{t+k+n}\right) \in$ $x^{k} \mathfrak{m}^{t} M$ for all $k, n \in \mathbf{N}$. Thus $f\left(\frac{1}{x^{n}}\right) \in \bigcap_{k} x^{k} \mathfrak{m}^{t} M=0$ for all $n \in \mathbf{N}$, by Krull Theorem. Therefore $f=0$.

THEOREM 2.10. Let $\mathfrak{a}$ be an ideal of a local ring $(R, \mathfrak{m})$ and $M$ a finitely generated $R$-module, and let $n \in \mathbf{N}$. Then the following statements are equivalent:

i) $\mathfrak{F}_{\mathfrak{a}}^{i}(M)$ is finitely generated for all $i>n$.

ii) $\operatorname{Coass}_{R}\left(\mathfrak{F}_{\mathfrak{a}}^{i}(M)\right) \subseteq\{\mathfrak{m}\}$ for all $i>n$.

iii) $\mathfrak{F}_{\mathfrak{a}}^{i}(M)=0$ for all $i>n$.

Proof. i) $\Rightarrow$ ii): By [8, Theorem 2.10].

ii) $\Rightarrow$ iii): Let $x \in \mathfrak{m} \backslash$ a. . Then by Lemma $2.9, \operatorname{Hom}_{R}\left(R_{x}, \mathfrak{F}_{\mathfrak{a}}^{i}(M)\right)=0$ for all $i>n$. But by [7, Theorem 3.15], there exists a long exact sequence

$$
\cdots \rightarrow \operatorname{Hom}_{R}\left(R_{x}, \mathfrak{F}_{\mathfrak{a}}^{i}(M)\right) \rightarrow \mathfrak{F}_{\mathfrak{a}}^{i}(M) \rightarrow \mathfrak{F}_{\langle\mathfrak{a}, x\rangle}^{i}(M) \rightarrow \operatorname{Hom}_{R}\left(R_{x}, \mathfrak{F}_{\mathfrak{a}}^{i+1}(M)\right) \rightarrow \cdots
$$


Hence we have $\mathfrak{F}_{\mathfrak{a}}^{i}(M) \simeq \mathfrak{F}_{\langle\mathfrak{a}, x\rangle}^{i}(M)$ for all $i>n$. Continuing in this way, we get $\mathfrak{F}_{\mathfrak{a}}^{i}(M) \simeq \mathfrak{F}_{\mathfrak{m}}^{i}(M)$ for all $i>n$. Since $\mathfrak{F}_{\mathfrak{m}}^{i}(M)=0$ for all $i \geq 0$, we get $\mathfrak{F}_{\mathfrak{a}}^{i}(M)=0$ for all $i>n$ and the proof is complete.

iii) $\Rightarrow \mathrm{i}$ ): It is clear.

The next result is a generalization of [1, Theorem 2.6].

Corollary 2.11. Let $\mathfrak{a}$ be an ideal of a local ring $(R, \mathfrak{m})$ and $M$ a finitely generated R-module. Let $l:=\operatorname{dim}(M / \mathfrak{a} M)$. Then $\operatorname{Coass}_{R} \mathfrak{F}_{\mathfrak{a}}^{l}(M) \nsubseteq\{\mathrm{m}\}$.

Proof. By [7, Theorem 4.5], $\mathfrak{F}_{\mathfrak{a}}^{i}(M)=0$ for all $i>l$ and $\mathfrak{F}_{\mathfrak{a}}^{l}(M) \neq 0$. If $\operatorname{Coass}_{R} \mathfrak{F}_{\mathfrak{a}}^{l}(M) \subseteq\{\mathfrak{m}\}$ then by the above theorem $\mathfrak{F}_{\mathfrak{a}}^{l}(M)=0$ which is a contradiction. Therefore the proof is complete.

Acknowledgment. The author would like to thank the referee for his/her useful suggestions.

\section{REFERENCES}

[ 1 ] M. Asgharzadeh and K. DivaAni-Aazar, Finiteness properties of formal local cohomology modules and Cohen-Macaulayness, Comm. Algebra 39 (2011), 1082-1103.

[2] M. H. BIJAN-ZADEH AND SH. REZAeI, Artinianness and attached primes of formal local cohomology modules, Algebra Colloquium 21 (2014), 307-316.

[ 3 ] M. BRodmanN AND R. Y. SHARP, Local cohomology: an algebraic introduction with geometric applications, Cambridge University Press, United Kingdom, 1998.

[4] N. Bourbaki, Commtative algebra, Addison-wesley, 1972.

[5] M. Eghbali, On Artinianness of formal local cohomology, colocalization and coassociated primes, Math. Scand. 113 (2013), 5-19.

[6] H. Matsumura, Commutative ring theory, Cambridge University Press, 1986.

[7] P. Schenzel, On formal local cohomology and connectedness, J. Algebra 315 (2007), 894-923.

[ 8 ] S. Yassemi, Coassociated primes, Comm. Algebra 23 (1995), 1473-1498.

[9] H. Zöschinger, Über koassoziierte Primideale, Math. Scand. 63 (1988), 196-211.

[10] H. Zöschinger, Minimax modules, J. Algebra 102 (1986), 1-32.

[11] H. ZöschiNGER, Über die Maximalbedingung für radikalvolle Untermoduln, Hokkaido Math. J. 17 (1988), 101-116.

Shahram Rezaei

PAyAme NoOR University (PNU)

Tehran

IRAN

E-mail: sha.rezaei@gmail.com 